\title{
PERSPECTIVE FORMAT FOR A PRIMARY FLIGHT DISPLAY AND ITS EFFECT ON PILOT SPATIAL AWARENESS
}

\author{
Nancy S. Dorighi, Arthur J. Grunwald, and Stephen R. Ellis \\ NASA Ames Research Center \\ Moffett Field, CA 94035-1000
}

\begin{abstract}
This paper describes an experimental study in progress which will evaluate pilot spatial awareness in low visibility conditions for two basic display types: (1) a computergenerated electronic attitude director indicator (EADI) within the conventional glass cockpit flight deck, and (2) a Tunnel-in-the-Sky perspective display used in place of the EADI. Pilot subjects navigate curved approach trajectories during instrument flight conditions (IFC). Their main task is to look in the direction (localize) where they would expect various targets to appear. The purpose of this study is to determine whether a perspective format such as the Tunnel Display can provide a significant improvement in pilot spatial awareness over conventional format in primary flight displays (PFDs).
\end{abstract}

\section{Introduction}

The volume of commercial aircraft is anticipated to increase into the next century, and it will require a system that allows closer spacing between aircraft, more fuel efficient trajectories, and reduced schedule disruptions due to poor visibility. Pilots may be challenged to manage narrower error tolerances than today, even in adverse weather conditions, and to navigate new curved approaches to runways made possible by MLS technology. To thus maximize efficient use of available airspace and airport facilities, yet ensure safety, perspective displays may help pilots maintain optimum spatial awareness.

Recent development in reliable on-board micro-processors and computer-generated image (CGI) technology has made possible a wealth of information never conceived of by the designers of the first flight deck instruments. Yet, the primary EADI display used in commercial aircraft today, continues to emulate the same gyroscopic device used in airplanes decades ago. Figures 1 and 2 illustrate the Boeing $757 \mathrm{ADI}$ and the analog horizon director from a circa 1960 s aircraft.

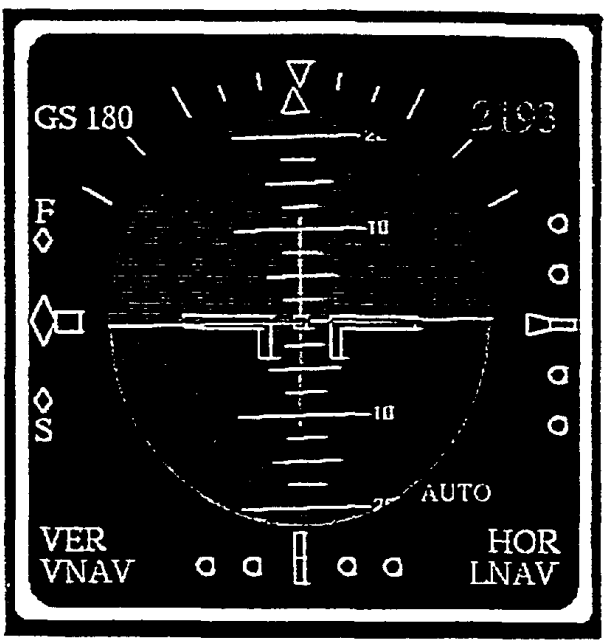

Figure 1. Boeing $757 \mathrm{ADI}$

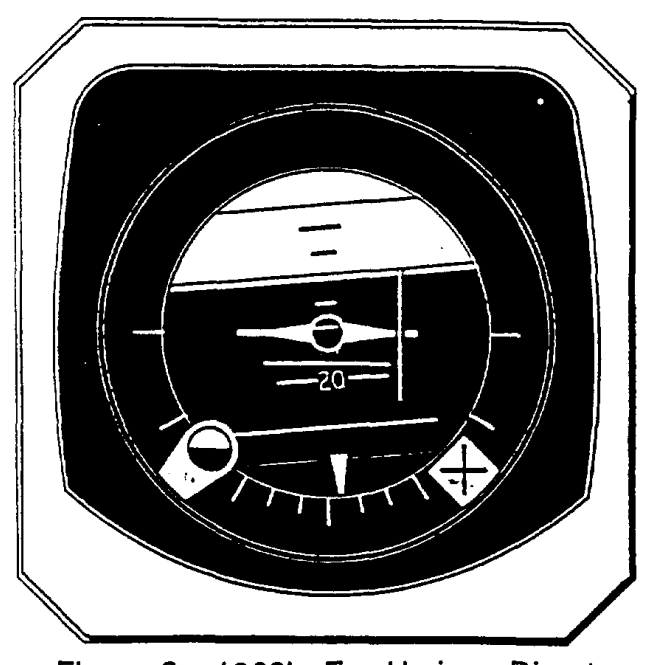

Figure 2. 1960's Era Horizon Director 
How Spatial Awareness Can Be Dearaded

The primary components of spatial orientation are a sense of one's location, velocity and direction with respect to fixed landmarks in the environment. The human vision system and vestibular system supply the inputs that allow us to make judgments of spatial orientation. Consequentially, insufficient or impaired sensory inputs in either or both of these areas impact overall spatial awareness. Ways to compensate for loss of spatial awareness in the cockpit are the use of maps, available displays or voice communication, but these may be insufficient in some cases.

In terms of the vision system, a glance out the window in good visibility tells pilots their location with respect to the terrain. it also provides the velocity vector by the apparent rate at which objects move in the visual field. Disorientation can be due to changes in heading or distraction during degraded visibility, and is responsible for many accidents. ${ }^{1}$ Inadvertent heading or altitude deviations are some of the consequences of pilots becoming distracted and disoriented.

In terms of the vestibular system, during long coordinated turns, the centripetal forces on the body combine with the lateral component of gravity. The sensed lateral acceleration is effectively zero, and with no visual cues there is little or no sense of turning. Under high workload, pilots may lose track of how far they have traveled through the turn. They may not have a very good idea of their orientation to the ground.

Under high workload or fatigue, the mental processing time for assimilating the information from the conventional flight deck, may be critically long. While the map display on the horizontal situation indicator (HSI) conveys the flight path in the horizontal plane, the pilot must look elsewhere to find the other information concerning his flight path orientation, for example, the glideslope and localizer deviations on the ADI.

Perspective Displays Can Enhance Spatial Awareness

Performance in pursuit tracking tasks such as the perspective display has been shown to be superior to compensatory tracking tasks such as the traditional EADI flight director. ${ }^{9}$ For orienting to the desired flight path, a perspective display can provide predictive information which conveys not only the future position of the aircraft, but also the future commanded path. Conventional flight directors compare predicted aircraft position and "current" commanded position for the cross-track deviation. However, spatial orientation may be improved when predicted position is compared to "predicted" commanded path because of the greater ability to anticipate (and correct) deviations. 10

A perspective display may be able to provide all the information available from a number of sources in the conventional cockpit, but in a more immediately usable format. ${ }^{7}$ Current glass cockpit instruments provide the horizontal (plan view) path given in the map display. To make a map display meaningful to the pilot's egocentric or body-referenced coordinate system, internal transformation is necessary which requires extra mental processing. A perspective ADI display may already be an egocentric display, and therefore enable more rapid comprehension of spatial orientation. Furthermore, integrating the information into a single display horizontal and vertical deviation, pitch and bank angle, terrain data, future path) may also improve comprehension speed. Information that can truly be assimilated "at a glance" would provide better spatial awareness. 2

Tunnel-in-the-Sky as a Candidate Perspective Display

It has been shown in previous work 4,5 that a Tunnel Display, furnished with adequate predictor information 6 is useful in particular for flying arbitrary curved MLS approaches. The Tunnel Display has been tested elaborately in the past on its merits of accuracy in following the curved path in the presence of turbulence, of entering the trajectory when initially randomly located outside the tunnel, and of failure detection in automated flight. The Tunnel Display was shown to outperform conventional instrumentation in curved approaches, in particular in the transitions from straight to curved sections, in its path 
entry characteristics and in providing a high degree of sensitivity to system failures. However, the most significant contribution of the Tunnel Display is the potential for increased pilot situational awareness.

A second merit of the display, when integrated properly in the Air Traffic Control Environment, is the ability of flexible and fast re-routing in flight. Fast re-routing might be vital in unforeseen hazardous situations, or for missed approaches. In these situations, Air Traffic Control might transfer the way points of the re-routed trajectory in an electronic format through a data link. The new tunnel would be generated instantaneously on-board, and the pilot would be visually presented with the requested change.

\section{Display Design}

The Tunnel-in-the-Sky is composed of a wire-frame image of the desired flight path and a predictor symbol, superimposed over basic terrain representations. See Figure 3. The wire-frame image (tunnel) has a constant square cross section $300 \mathrm{ft}$, and dashed corner lines. Two tunnel cross sections are constantly displayed 3.5 and 7 seconds ahead.
The four sides have rectangular cross-sections which show the allowable vertical and lateral deviations. The visual field is, like the ADI, inside-out, analogous to looking through a aircraft windshield, and has a field of view of $90^{\circ}$ horizontally and vertically. The wireframe structure of the tunnel enables seethrough to the terrain and horizon. The dashed corner lines are made up of $200 \mathrm{ft}$ straight segments, which connect the corners of the cross sections. These corner lines are displayed for the entire length of the tunnel, thereby defining the future path.

Superimposed on the tunnel image is a symbol which represents the aircraft's position predicted 7 seconds ahead. The symbol fits inside the far cross section and remains upright at all times. The cross represents the future "actual" position while the square the future "commanded" position. Bringing the cross inside the square constitutes a useful control law which equals a well-designed quickened flight director.

The tunnel with its predictor symbol are superimposed over simple stylistic computergenerated terrain images which depict the immediate airport area of about 500 square miles. These include flat ground overlaid with

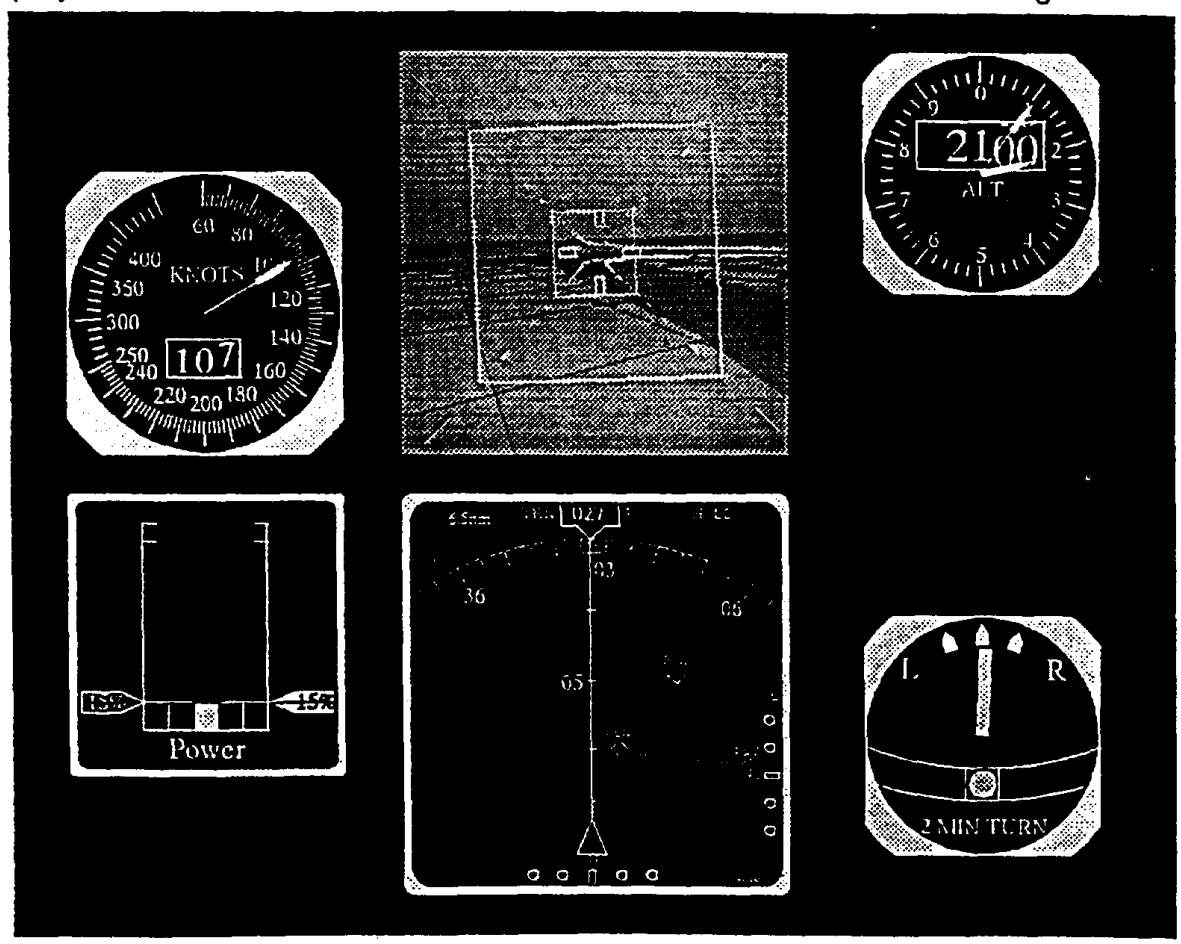

Figure 3. Tunnel-in-the-Sky Display Replacing ADI in B757 Main Instrument Panel 
a North-South oriented grid, bodies of water, tall structures, etc. The runways show the runway numbers, center line, threshold markings, and approach lights.

The Tunnel-in-the-Sky is displayed in place of the conventional EADI (Electronic Attitude Direction Indicator) in the conventional display set described below.

For the experimental control display, an emulation of current glass transport instruments is used. See Figure 4. The EADI depicts ground speed, altitude, pitch reference lines, flight director roll and pitch command needles, roll pointer, and vertical and horizontal error bugs. Directoy below the EADI is the HSI display in map mode which shows the horizontal trajectory, compass row arc with heading bug, ownship symbol, trend vector arcs, vertical and horizontal flight path errors, and target symbols. Other instruments include Airspeed Indicator, Power Meter, Altimeter and Turn Coordinator. In this study an autothrottle maintains speed at 130 knots and a turn coordinator controls the sideslip. The approximate dynamics of a Boeing 727 were modeled for the aircraft.

\section{Method}

Experienced commercial pilots familiar with the New York City area have been recruited for this study. The experiments are conducted inside a darkened $1.5 \mathrm{~m}$ radius dome which has been used for previous situational awareness experiments with perspective displays. ${ }^{3}$ See Figure 5. The displays are shown on an IRIS 4D 210/GTXB graphics color monitor placed at the center of the dome. Subjects are seated in a chair at a distance of about $100 \mathrm{~cm}$ from the monitor with a downward viewing angle of $35^{\circ}$, and a field of view of $8.5^{\circ}$. A headmounted light pointer is used to feedback direction by projecting a light inside the dome. A calibrated Polhemus electromagnetic head. tracker measures head position and reports angles in azimuth and elevation and roll.

A hand controller (Measurement Systems Model 547 Three Axis Joystick) is mounted on the right arm of the chair and is used to control pitch and roll. Three pushbuttons and a trigger button on the controller allow the subjects to start or abort an approach, signal data to be taken, and toggle between autopilot and manual control.

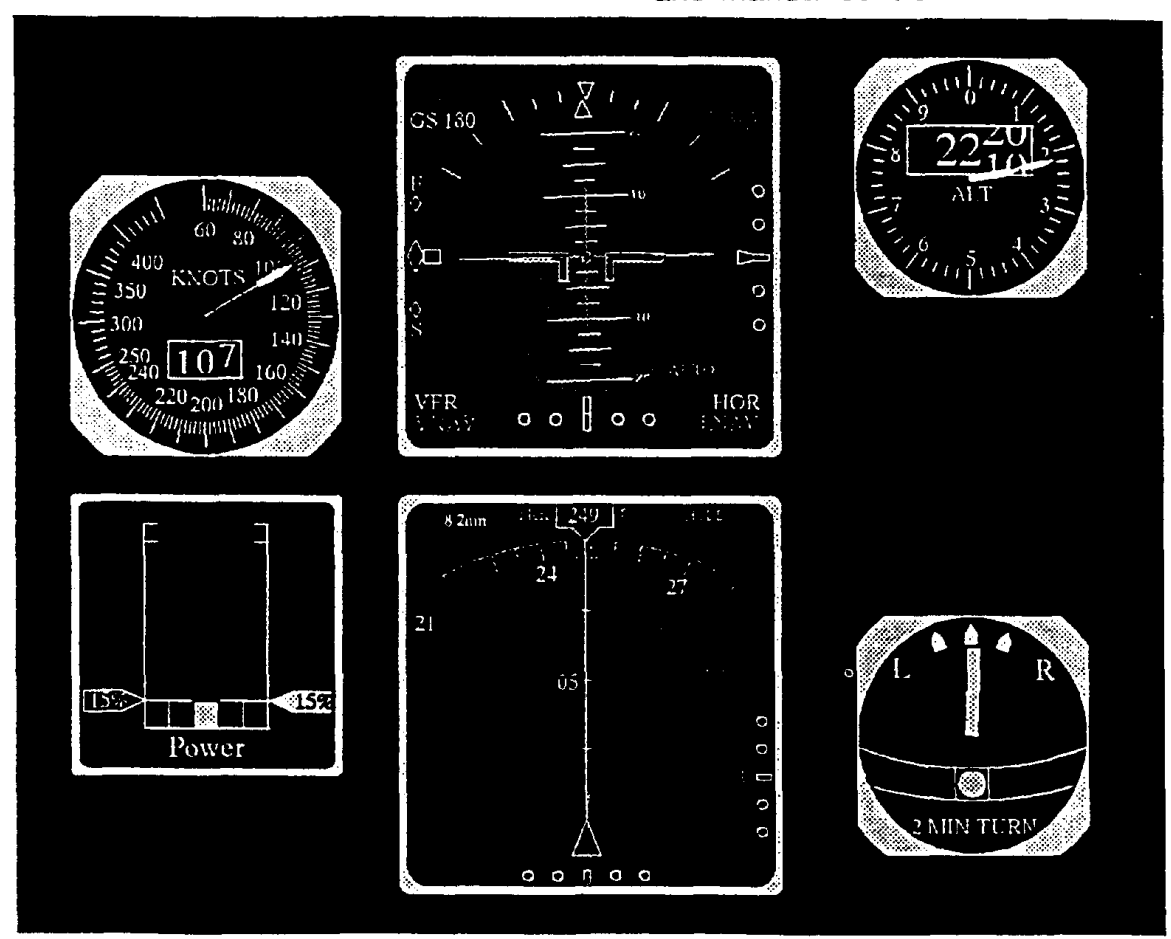

Figure 4. B757 Main Instrument Panel 


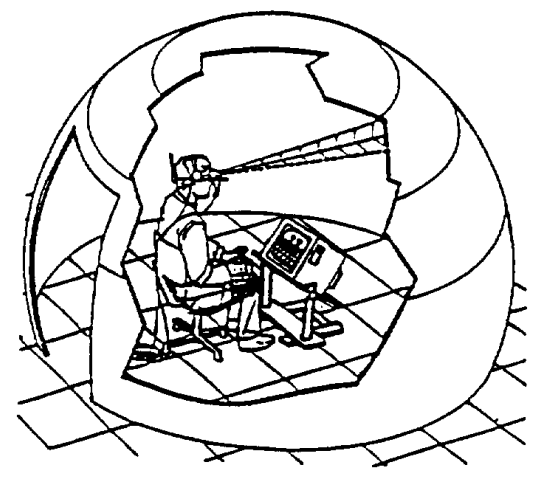

Figure 5. Pilot Subject in Dome Wearing Head Tracker

During pre-trial training, pilots are asked to familiarize themselves with the five different curved approaches to La Guardia airport. The approaches were developed by the FAA for possible future MLS (Microwave Landing System) implementation. See Figure 6. They are also asked to memorize the location of seven different targets that might be relevant ground references for an aircraft on final approach. These are the World Trade Center, the George Washington Bridge, the Maspeth Tanks, Shea Stadium, the Whitestone Bridge, the Runway, and the Bronx Park. See Figure 6 . Preliminary measurements are taken of subject's basic error in estimating angles by asking them to gaze in random repetitions of angles from the 9 o'clock to 3 o'clock position.

Subjects fly six repetitions of each of the five approaches to La Guardia, in a carefully arranged random order. At the start of each run the aircraft is positioned at an altitude of approximately $2500 \mathrm{ft}$. and 10 miles from touchdown, and will consist of one or two moderate turns (10 degrees maximum bank angle) and a final straight segment which ends just before touchdown. On one of the five approaches, the pilot will be instructed to execute a missed approach.

The pilot subjects were divided into four groups. Two groups fly the tunnel display, and two fly the current transport glass instrument display. Within each display type, one group performs the primary task of monitoring the approaches using the autopilot, and the other flies manually. Cross-winds have been added to enhance the workload. The control task is difficult enough that if left for several seconds, the aircraft would drift off the required trajectory.

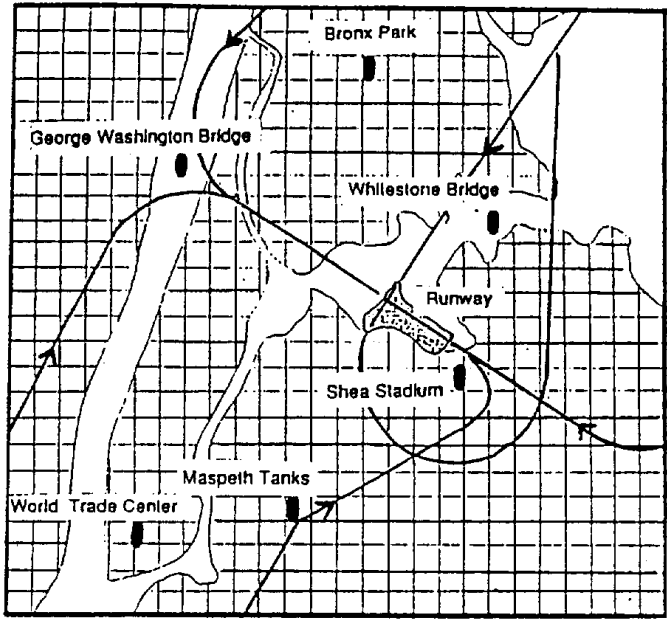

Figure 6. Curved Approach Profiles and Targets in the LGA Area

An IBM PC/AT, serially connected to the host computer, plays back upon command from the IRIS, prerecorded audio messages which at predefined points in the approach, convey clearances or instructions to the pilot that would be provided by controllers. In addition, some messages ask the pilots to localize one of the seven targets which are in the environment. They are asked to rotate their head to look in the direction of the indicated reference as quickly and accurately as possible. The number of directional queries are randomly selected for each approach, and varies from four to six, to avoid subjects anticipating localizations or associating them with approaches.

Measurements are taken of the azimuth and elevation deviation of the perceived direction from the true direction, as well as the time taken by the subject to make the determination. A time-history of the azimuth and elevation angles of the gaze direction is also recorded. The approach continues to touchdown. 
At the conclusion of the session, the pilots complete a questionnaire which asks for a subjective evaluation of the display regarding ease of use and effectiveness for keeping track of spatial orientation.

\section{Evaluation Plan}

It is hypothesized that, by integrating future path and bank angle with basic ground references into a single display, the perspective displays may provide pilots with more spatial information in a more quickly comprehended format. This would be most evident when ground references are not available out the window. 3 Thus able to assess their spatial orientation more frequently, pilots would be better informed of their overall situation. In this way this display concept may compensate in the absence of other visual cues, and thus help avoid disorientation that is an underlying cause of many mishaps today. 8

Pilot spatial awareness will be assessed by measuring localization error and response time in pilot judgments. Confidence in the judgments will be evaluated from the time histories of the head position and from subjective self-evaluation of the subjects. Comparisons will be made of measurements taken during periods of distraction, higher workload, straight trajectory, and during the first and second half of turns.

If significant improvements in spatial awareness with the perspective display are indicated in the experiment, further study in a realistic cockpit simulation will be planned as a follow-on to this research.

\section{Acknowledgments}

Russ Parrish, NASA/Langley Flight Management Division, Crew/Vehicle Interface Research Branch for the conventional instrument implementation.
Barry Scott, FAA, for the curved approach definitions.

\section{References}

1 Bennett, C.T. and Schwirzke, M., "Instrument Approach Accident Analysis," Proceedings of the 34th Annual Meeting of the Human Factors Society, Nov. 1990.

${ }^{2}$ Ellis, S.E., McGreevy, M. W., and Hitchcock, R.J., "Perspective Traffic Display Format and Airline Pilot Traffic Avoidance," Human Factors, Vol. 29(4), Aug. 1987, pp.371-382.

3 Ellis, S.E., Smith, S., and Hacisalinzade, S.,"Visual Direction as a Metric of Virtual Space," Proceedings of the 33rd Annual Meeting of the Human Factors Society, Oct. 1989.

4 Grunwald, A.J., Robertson, J.B. and Hatfield, J.J., "Experimental Evaluation of a Perspective Tunnel Display for ThreeDimensional Helicopter Approaches," AIAA Journal of Guidance and Control, Vol. 4, No. 6, Nov.-Dec. 1981, pp. 623-631.

5 Grunwald, A.J., "Tunnel Display for Four-dimensional Fixed-Wing Approaches," Journal of Guidance, Control and Dynamics, Vol. 7, May-June 1984, pp. 369-377.

6 Grunwald, A.J., "Predictor Laws for Pictorial Flight Displays", AIAA Journal of Guidance, Control and Dynamics, Vol. 8, No. 5, Sept.-Oct. 1985, pp. 545-552

7 Larkin, J.H., and Simon, H.A., "Why a Diagram Is (Sometimes) Worth Ten Thousand Words," Cognitive Science, Vol. 11, 1987, pp. 65-99.

8 National Transportation Safety Board, "Spatial Disorientation Involving Weather," Database search of accidents between Jan. 1983 and July 1989.

9 Poulton, E.C., "Tracking Skill and Manual Control," New York: Academic Press, 1974.

10 Stokes, A., Wickens, C. and Kite, K., "Display Technology, Human Factors Concepts," Society of Automotive Engineers, Inc., 1990. 